\title{
Facile Synthesis of Some Novel \\ Pyrido[3', 2': 4, 5]thieno[2,3-b][1,4]thiazine-8-carboxylic Acids
}

\author{
Mohammed H. Al-Huniti ${ }^{1}$, Mustafa M. El-Abadelah ${ }^{1}$, Jalal A. Zahra ${ }^{1, *}$, Salim S. Sabri ${ }^{2}$ and \\ Arnd Ingendoh ${ }^{3}$
}

${ }^{1}$ Chemistry Department, Faculty of Science, The University of Jordan, Amman 11942, Jordan; Emails:mmharb@ju.edu.jo,mustelab@ju.edu.jo

${ }^{2}$ University of Sharjah, Sharjah, P. O. Box 27272, United Arab Emirates (UAE); E-mail: salim@sharjah.ac.ae

${ }^{3}$ Bruker Daltonik GmbH, Fahrenheitstr. 4, D-28359 Bremen, Germany; E-mail: ai@bdal.de

*Author to whom correspondence should be addressed; E-mail: Zahra@ju.edu.jo; Fax: +962-6 5348932; Tel.: +962-6 5355000 (ext. 22163)

Received: 8 March 2007; in revised form: 14 March 2007 / Accepted: 15 March 2007 / Published: 15 March 2007

\begin{abstract}
Model tetrahydropyrido[3',2':4,5]thieno[2,3-b][1,4]thiazines 9a-c were synthesized via reductive lactamization, using sodium dithionite, of the respective 2[(carboxyalkyl)thio]-3-nitro-4,7-dihydrothieno[2,3-b]pyridine-5-carboxylic acids 7a-c. The latter derivatives were made via interaction of 2-chloro-7-cyclopropyl-3-nitro-4,7dihydrothieno[2,3-b]pyridine-5-carboxylic acid (6) with each of $\alpha$-mercaptoacetic, $\alpha$ mercaptopropionic, and $\alpha$-mercaptosuccinic acids and triethylamine in aqueous acetone at room temperature. The structures of $\mathbf{7 a - 7} \mathbf{c}$ and $\mathbf{9 a - 9 c}$ are supported by microanalytical and spectral (IR, MS, NMR) data. Compounds 9a and 9c showed potent inhibitory activity against the IGROV1 (Ovarian Cancer) cell line.
\end{abstract}

Keywords: 2-Chloro-7-cyclopropyl-3-nitro-4-oxothieno[2,3-b]pyridine-5-carboxylic acid, $\alpha$-mercaptoalkanoic acids, $\mathrm{S}_{\mathrm{N}^{-}}$-Ar reaction, reductive lactamization, tetrahydropyrido[3' ,2':4,5]thieno[2,3-b][1,4] thiazines, antitumor activity. 


\section{Introduction}

Several substituted 4-oxothieno[2,3-b]pyridine-5-carboxylic acids (exemplified by 1 [1], Figure 1) bioisosteres of fluoroquinolone antibacterials (such as ciprofloxacin), were synthesized and reported to exhibit "good to excellent" levels of antibacterial potency [1-4]. On the other hand, thieno[2,3-b][1,4]thiazine derivatives (e.g. 2, Figure 1) are currently of interest due to their as therapeutic properties as smooth muscle relaxants [5] and as potassium channel-opening agents [6] which make them potentially useful for the treatment of various diseases, while certain thieno[2,3-b][1,4]thiazine-2-ones (e.g. 3) have been patented as urokinase inhibitors [7].

Figure 1. Structures of 4-oxothieno[2,3-b]pyridine (1) and thieno[2,3-b][1,4]thiazines 2, $\mathbf{3}$.

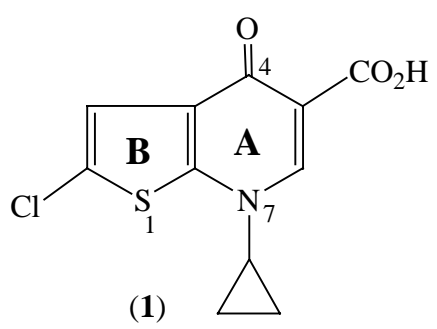

(1)

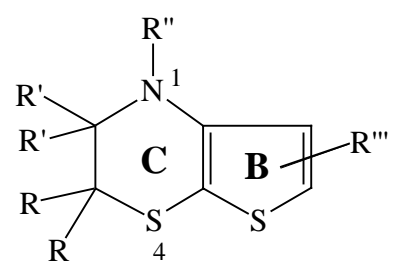

(2)

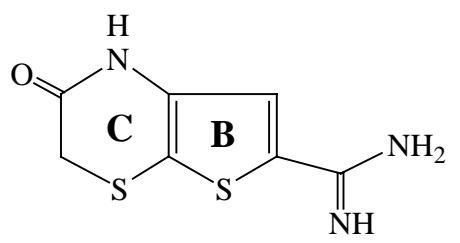

(3)

We became interested in condensed pyridothieno[1,4]thiazine tricyclic systems, for which literature data are confined to only one report [8] describing the preparation and properties of pyrido[2,1-c]thieno[3,2-e][1,4]thiazines 4 and their dihydro precursors 5 (Figure 2).

Figure 2. Structures of pyrido[2,1-c]thieno[3,2-e][1,4]thiazines 4, 5.<smiles>[R]C1=C2C(C)=CC(C)=CN2c2c(sc(C(=O)OCC)c2[R])S1</smiles>

(4)<smiles>[R]c1c(C(=O)OCC)sc2c1N1C=C(C)C=C(C)[C@]1([2H])[C@H]([R2])S2</smiles>

(5)

In particular, we envisaged that the hitherto undescribed tetrahydro- $1 H$-pyrido[3',2':4,5]thieno[2,3b][1,4]thiazine-8-carboxylic acids 9a-9c (Scheme 1), representing a tricyclic hybrid of $\mathbf{1}$ and $\mathbf{3}$, might exhibit interesting bioproperties such as antimicrobial and antitumor activity. Hence, the present work deals with the synthesis and properties of 9a-9c, as outlined in Scheme 1 and detailed in the Experimental section. These novel heterocyclics 9a-9c are potential bioisosteres of the recently described [1,4]thiazino-[2,3-h]quinoline-8-carboxylic acids 10a-10c shown in Scheme 1 [9], in which the benzene nucleus $(\mathbf{B})$ is replaced by a thiophene ring. 
Scheme 1. Synthesis of pyrido[3', 2': 4, 5]thieno[2,3-b][1,4]thiazines 9a-9c.<smiles>CC(C)C(=O)c1cn(C2CC2)c2sc(Cl)c([N+](=O)[O-])c2c1=O</smiles>

(6)<smiles>[R]C1Sc2c(F)cc3c(=O)c(C(=O)O)cn(C4CC4)c3c2NC1=O</smiles><smiles>[R]C(Sc1sc2c(c1[N+](=O)[O-])c(=O)c(C(=O)O)cn2C1CC1)C(=O)O</smiles>

(7a-7c)

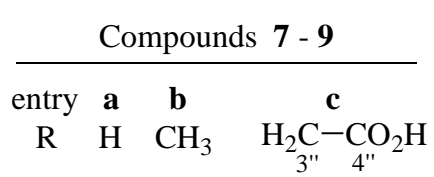

Reagents and Conditions:

(i) $\mathrm{HS}-\mathrm{CH}(\mathrm{R}) \mathrm{CO}_{2} \mathrm{H} /$ aq. acetone, $\mathrm{NEt}_{3}$

(ii) $\mathrm{Na}_{2} \mathrm{~S}_{2} \mathrm{O}_{4}$, aq. $\mathrm{K}_{2} \mathrm{CO}_{3} / \mathrm{rt}$

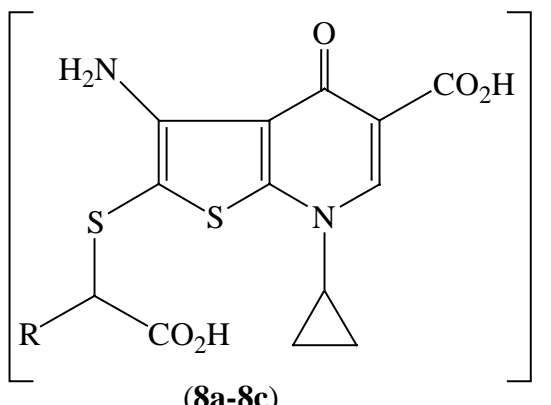

(8a-8c)

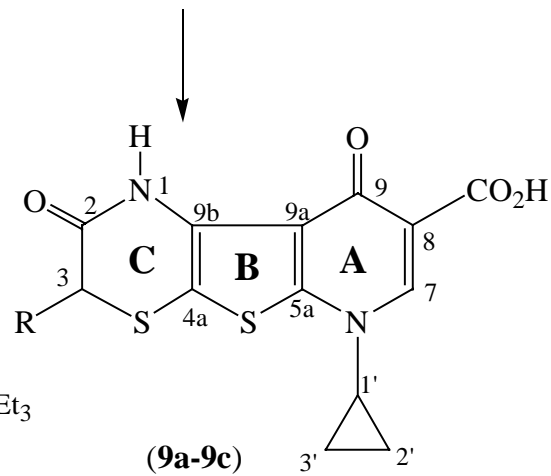

\section{Results and Discussion}

The synthesis of compounds 9a-9c is achieved by utilizing 2-chloro-7-cyclopropyl-3-nitro-4-oxo4,7-dihydrothieno[2,3-b]pyridine-5-carboxylic acid (6) [2] as a common synthon, and constructing the thiazinone nucleus thereon through two-step conversions as illustrated in Scheme 1. The first step entails the preparation of the acyclic precursors 7a-7c via direct interaction of the appropriate $\alpha-$ mercapto-alkanoic acids with the synthon $\mathbf{6}$ in aqueous acetone containing triethylamine. This reaction follows an $\mathrm{S}_{\mathrm{N}}-\mathrm{Ar}$ (addition-elimination) path, and is facilitated by the presence of the electronwithdrawing $\mathrm{C}(4)$-keto and the $\mathrm{C}(3)$-nitro groups. Reduction of 7a-7c with sodium dithionite in aqueous potassium carbonate converts the nitro group to an amino group, and is followed by spontaneous lactamization of the resultant 8-amino intermediates 8a-8c to afford good yields of the corresponding target products 9a-9c in fairly pure form. The required common synthon $\mathbf{6}$ is made accessible by nitration of $\mathbf{1}$, which in turn is prepared from 3-acetyl-2,5-dichlorothiophene according to a literature procedure [1].

\section{Antitumor Activity}

Compounds 9 and 10 were tested using $10 \mu \mathrm{M}$ concentration against the panel of 60 human cancer cell lines used by the National Cancer Institute (NCI, USA). The most affected cell line was IGROV1 (from Ovarian Cancer). The percentage growth inhibitions at $10 \mu \mathrm{M}$ were $76 \%, 64 \%$, 65\% and 88\% for compounds 9a, 9c, 10a and 10c, respectively. 


\section{Experimental}

\section{General}

2,5-Dichlorothiophene, ethyl 3-( $N, N$-dimethylamino)acrylate and cyclopropylamine were purchased from Acros. ( \pm )-2-Mercaptopropionic acid, ( \pm )-2-mercaptosuccinic acid and mercaptoacetic acid were purchased from Aldrich. Melting points were determined on a Gallenkamp capillary melting point apparatus and are uncorrected. ${ }^{1} \mathrm{H}$ - and ${ }^{13} \mathrm{C}$-NMR spectra were measured on a Bruker DPX-300 instrument with $\mathrm{Me}_{4} \mathrm{Si}$ as internal reference. High resolution mass spectra (HRMS) were measured in positive ion mode by Electrospray (ESI) on APEX-Qe 94 instrument. The samples were dissolved in acetonitrile, diluted in spray solution (methanol/water $1: 1 \mathrm{v} / \mathrm{v}+0.1 \%$ formic acid) and infused using a syringe pump with a flow of $2 \mathrm{uL} / \mathrm{min}$. External calibration was conducted using Arginine cluster in a mass range $\mathrm{m} / \mathrm{z}$ 175-871. MS/MS spectra for 7a and $\mathbf{7 b}$ were performed in the external Qh of the APEX-Q. For all HRMS data, the mass error was 0.00-0.50 ppm. IR spectra were recorded as $\mathrm{KBr}$ discs on a Nicolet Impact-400 FT-IR spectrophotometer. Elemental analyses were preformed at the Microanalytical Laboratory of the Hashemite University, Zarqa, Jordan.

\section{2-[(Carboxymethyl)thio]-7-cyclopropyl-3-nitro-4-oxo-4,7-dihydrothieno[2,3-b]pyridine-5-carboxylic acid (7a).}

Mercaptoacetic acid (0.46 g, $5 \mathrm{mmol}$ ) was added to a stirred solution of 2-chloro-7-cyclopropyl-3nitro-4-oxo-4,7-dihydrothieno[2,3-b]pyridine-5-carboxylic acid (6, $1.32 \mathrm{~g}, 4.2 \mathrm{mmol}$ ) in aqueous acetone $(1: 2 \mathrm{v} / \mathrm{v}, 54 \mathrm{~mL})$ and triethylamine $(6 \mathrm{~mL})$ at $\mathrm{rt}$, and then kept in the dark for $7 \mathrm{~h}$. The reaction mixture was then washed with chloroform $(2 \times 10 \mathrm{~mL})$, the aqueous layer was acidified with $3 \mathrm{~N} \mathrm{HCl}$ and the precipitated product was collected and dried. The title compound was purified by stirring in boiling chloroform $(10 \mathrm{~mL})$ in which the soluble impurities are removed. Yield $1.37 \mathrm{~g}$ (88 \%); mp 236-237 ${ }^{\circ} \mathrm{C}$ (decomp); IR ( $\mathrm{cm}^{-1}$ ) 3601, 3529, 3408, 3016, 2932, 1744, 1728, 1695, 1615, 1525, 1502, 1479, 1426, 1337, 1236, 1183; ${ }^{1} \mathrm{H}-\mathrm{NMR}$ (300 MHz, DMSO-d 6 ) $\delta 1.16 / 1.29$ (2 m, 4H, 2H-2' / 2H-3'), 3.85 (m, 1H, H-1'), 4.06 (s, 2H, 2H-2"), 8.61 (s, 1H, H-6), 13.28 [br s, $1 \mathrm{H}, \mathrm{C}\left(2^{\prime \prime}\right)-\mathrm{CO}_{2} \mathrm{H}$ ], 14.56 [br s, 1H, C(5)-CO $\left.{ }_{2} H\right]$; ${ }^{13} \mathrm{C}-\mathrm{NMR}$ (75 MHz, DMSO-d 6 ) $\delta 7.8$ (C-2'/C-3'), 38.2 (C-2"), 38.9 (C-1'), 113.0 (C5), 120.3 (C-3), 133.7 (C-2), 142.3 (C-3a), 147.0 (C-6), 152.8 (C-7a), 165.4 [C(5)-CO $\mathrm{CO}_{2} \mathrm{H}$ ], 169.9 [C(1")-CO $\left.\mathrm{CO}_{2} \mathrm{H}\right], 171.9$ (C-4); HRMS: calcd. for $\mathrm{C}_{13} \mathrm{H}_{11} \mathrm{~N}_{2} \mathrm{O}_{7} \mathrm{~S}_{2}{ }^{+}[\mathrm{M}+\mathrm{H}]^{+}$: 371.00077, found: 371.00040; calcd. for $\mathrm{C}_{13} \mathrm{H}_{10} \mathrm{~N}_{2} \mathrm{O}_{7} \mathrm{~S}_{2} \mathrm{Na}^{+}[\mathrm{M}+\mathrm{Na}]^{+}$: 392. 98271, found: 392.98237; MS/MS (of m/z 371): $\mathrm{C}_{8} \mathrm{H}_{6} \mathrm{NS}_{2}{ }^{+}$ (179.99367), $\mathrm{C}_{11} \mathrm{H}_{6} \mathrm{NO}_{2} \mathrm{~S}_{2}^{+}$(247.98351), $\mathrm{C}_{8} \mathrm{H}_{3} \mathrm{~N}_{2} \mathrm{O}_{4} \mathrm{~S}_{2}^{+}$(255.95301); Anal. calcd. for $\mathrm{C}_{13} \mathrm{H}_{10} \mathrm{~N}_{2} \mathrm{O}_{7} \mathrm{~S}_{2}$ (370.36): C, 42.16; H, 2.72; N, 7.56; S, 17.32. Found: C, 41.78; H, 2.93; N, 7.60; S, 17.50.

2-[(Carboxyethyl)thio]-7-cyclopropyl-3-nitro-4-oxo-4,7-dihydrothieno[2,3-b]pyridine-5-carboxylic acid $[( \pm)-7 \mathbf{b}]$

Prepared from ( \pm )-2-mercaptopropionic acid (0.53 g, $5 \mathrm{mmol}$ ) and 6 (1.32 g, $4.2 \mathrm{mmol})$ using the procedure and experimental conditions described above for the preparation of 7a. The title compound was isolated as a yellow solid which was recrystallized from chloroform. Yield $1.26 \mathrm{~g}$ (78 \%); mp 221$222^{\circ} \mathrm{C}$ (decomp); IR (cm ${ }^{-1}$ ) 3427, 3205, 3106, 3078, 3003, 2925, 1736, 1691, 1602, 1546, 1470, 1335, 
1294, 1215, 1182; ${ }^{1} \mathrm{H}-\mathrm{NMR}$ (300 MHz, DMSO-d 6 ) $\delta 1.17 / 1.31$ (m, 4H, 2H-2'/2H-3'), 1.40 (d, $J=7.1$ Hz, 3H, $\mathrm{CH}_{3}$ ), 3.87 (m, 1H, H-1'), 4.10 (q, $J=7.1 \mathrm{~Hz}, 1 \mathrm{H}, \mathrm{H}-2^{\prime \prime}$ ), 8.64 (s, 1H, H-6), 13.34 [br s, 1H, $\mathrm{C}\left(2^{\prime \prime}\right)-\mathrm{CO}_{2} \mathrm{H}$ ], 14.40 [br s, $1 \mathrm{H}, \mathrm{C}(5)-\mathrm{CO}_{2} \mathrm{H}$ ]; ${ }^{13} \mathrm{C}-\mathrm{NMR}$ (75 MHz, DMSO-d 6 ) $\delta 7.7$ (C-2'/C3'), 18.0 ( $\mathrm{CH}_{3}$ ), 38.3 (C-1'), 47.9 (C-2"), 112.9 (C-5), 120.1 (C-3), 126.6 (C-2), 145.6 (C-3a), 147.6 (C-6), 154.2 (C-7a), 165.2 [C(5)- $\mathrm{CO}_{2} \mathrm{H}$ ], 172.1 (C-4), 172.5 [C(1")- $\mathrm{CO}_{2} \mathrm{H}$ ]; HRMS: calcd. for $\mathrm{C}_{14} \mathrm{H}_{13} \mathrm{~N}_{2} \mathrm{O}_{7} \mathrm{~S}_{2}{ }^{+}$ $[\mathrm{M}+\mathrm{H}]^{+}$: 385.01642, found: 385.01592; calcd. for $\mathrm{C}_{14} \mathrm{H}_{12} \mathrm{~N}_{2} \mathrm{O}_{7} \mathrm{~S}_{2} \mathrm{Na}^{+}[\mathrm{M}+\mathrm{Na}]^{+}$: 406.99836, found: 406.99781. MS/MS (of m/z 385): $\mathrm{C}_{8} \mathrm{H}_{6} \mathrm{NS}_{2}{ }^{+}$(179.99363), $\mathrm{C}_{11} \mathrm{H}_{6} \mathrm{NO}_{2} \mathrm{~S}_{2}{ }^{+}$(247.98347), $\mathrm{C}_{8} \mathrm{H}_{3} \mathrm{~N}_{2} \mathrm{O}_{4} \mathrm{~S}_{2}{ }^{+}$ (255.95303), $\mathrm{C}_{11} \mathrm{H}_{9} \mathrm{NO}_{3} \mathrm{~S}_{2}^{+}$(267.00185), $\mathrm{C}_{11} \mathrm{H}_{8} \mathrm{~N}_{2} \mathrm{O}_{5} \mathrm{~S}_{2}{ }^{+}$(311.98696); Anal. calcd. for $\mathrm{C}_{14} \mathrm{H}_{12} \mathrm{~N}_{2} \mathrm{O}_{7} \mathrm{~S}_{2}$ (384.39): C, 43.74; H, 3.15; N, 7.29; S, 16.68. Found: C, 43.63; H, 3.14; N, 7.04; S, 16.66.

2-[(Carboxyl-7-cyclopropyl-3-nitro-4-oxo-4,7-dihydrothieno[2,3-b]pyridin-2-yl)thio]succinic acid $[( \pm)-7 c]$

Prepared from ( \pm )-2-mercaptosuccinic acid $(0.75 \mathrm{~g}, 5 \mathrm{mmol})$ and 6 (1.32 g, $4.2 \mathrm{mmol})$ using the procedure and experimental conditions described above for the preparation of $7 \mathbf{a}$. The title compound was obtained as a yellow precipitate which was collected, washed successively with chloroform and methanol and dried. Yield 1.62 g (90\%); mp 211-212 ${ }^{\circ} \mathrm{C}$ (decomp); IR (cm $\left.{ }^{-1}\right) 3440$ (br), 3092, 2928, 1724 (br), 1708, 1596, 1529, 1501, 1454, 1416, 1376, 1325, 1260, 1178; ${ }^{1} \mathrm{H}-\mathrm{NMR}$ (300MHz, DMSO$\left.\mathrm{d}_{6}\right) \delta 1.17 / 1.31$ (2 m, 4H, 2H-2' / 2H-3'), 2.74 (dd, $\left.J=16.8,6.2 \mathrm{~Hz}, 1 \mathrm{H}, \mathrm{H}_{\mathrm{A}}-3^{\prime \prime}\right), 2.81$ (dd, $J=16.8,7.7$ $\mathrm{Hz}, 1 \mathrm{H}, \mathrm{H}_{\mathrm{B}}-3^{\prime \prime}$ ), 3.87 (m, 1H, H-1'), 4.15 (dd, $J=6.2,7.7 \mathrm{~Hz}, 1 \mathrm{H}, \mathrm{H}-2^{\prime \prime}$ ), 8.68 (s, 1H, H-6), 13.05 [br s, $2 \mathrm{H}, 2 \mathrm{CO}_{2} \mathrm{H}$ ], 14.38 [br s, $1 \mathrm{H}, \mathrm{C}(5)-\mathrm{CO}_{2} \mathrm{H}$ ]; ${ }^{13} \mathrm{C}-\mathrm{NMR}$ (75 MHz, DMSO-d 6 ) $\delta 7.8\left(\mathrm{C}-2^{\prime} / \mathrm{C}-3\right.$ '), $36.5(\mathrm{C}-$ 3"), 38.4 ( C-1'), 48.4 (C-2"), 112.9 (C-5), 120.0 (C-3), 126.5 (C-2), 145.6 (C-3a), 147.7 (C-6), 154.3 (C-7a), 165.2 [C(5)-CO ${ }_{2} \mathrm{H}$ ], 171.3 [C(1")- $\mathrm{CO}_{2} \mathrm{H}$ ], 171.8 [C(4")-CO $\mathrm{CO}_{2} \mathrm{H}$ ], 172.1 (C-4); HRMS: calcd. for $\mathrm{C}_{15} \mathrm{H}_{11} \mathrm{~N}_{2} \mathrm{O}_{9} \mathrm{~S}_{2}{ }^{+}[\mathrm{M}-\mathrm{H}]^{+}:$426.99060, found: 426.99128; Anal. calcd. for $\mathrm{C}_{15} \mathrm{H}_{12} \mathrm{~N}_{2} \mathrm{O}_{9} \mathrm{~S}_{2}$ (428.40): C, 42.05; H, 2.82; N, 6.54; S, 14.97. Found: C, 42.17; H, 3.01; N, 6.60; S, 15.06.

6-Cyclopropyl-2,9-dioxo-2,3,6,9-tetrahydro-1H-pyrido[3',2':4,5] thieno[2,3-b][1,4] thiazine-8carboxylic acid (9a)

To a vigorously stirred suspension of 2-[(carboxymethyl)thio]-7-cyclopropyl-3-nitro-4-oxo-4,7dihydrothieno[2,3-b]pyridine-5-carboxylic acid (7a, $0.37 \mathrm{~g}, 1 \mathrm{mmol}$ ) in concentrated hydrochloric acid (12 $\mathrm{mL}$ ) was added, portionwise, stannous chloride dihydrate ( $1.13 \mathrm{~g}, 5 \mathrm{mmol})$. The mixture was stirred for $1 \mathrm{~h}$, then treated with water $(12 \mathrm{~mL})$ and was kept under stirring at rt until a clear solution was obtained. This solution was finally neutralized with aqueous sodium carbonate whereby a deep brown precipitate was formed, which was collected, washed with cold water, cold ethanol and dried. Yield 0.27 g (84 \%); mp 274-275 ${ }^{\circ} \mathrm{C}$ (decomp); IR (cm $\left.{ }^{-1}\right) 3440$ (br), 3343, 3080, 3009, 2913, 1719, 1691, 1614, 1543, 1460, 1440, 1337, 1228, 1177; ${ }^{1} \mathrm{H}-\mathrm{NMR}$ (300 MHz, DMSO-d 6 ) $\delta 1.15 / 1.26$ (2 m, 4H, 2H-2' / 2H-3'), 3.64 (s, 2H, 2H-3), 3.82 (m, 1H, H-1'), 8.54 (s, 1H, H-7), 9.52 (s, 1H, H-1), 14.61 (br s, $\left.1 \mathrm{H}, \mathrm{CO}_{2} \mathrm{H}\right) ;{ }^{13} \mathrm{C}-\mathrm{NMR}\left(75 \mathrm{MHz}, \mathrm{DMSO}^{-\mathrm{d}_{6}}\right) \delta 7.5$ (C-2'/C-3'), 30.7 (C-3), 38.2 (C-1'), 106.6 (C4a), 111.9 (C-9b), 119.8 (C-8), 132.6 (C-9a), 145.9 (C-7),151.9 (C-5a), 163.4 (C-2), $165.7\left(\mathrm{CO}_{2} \mathrm{H}\right)$, 173.7 (C-9); HRMS: calcd. for $\mathrm{C}_{13} \mathrm{H}_{11} \mathrm{~N}_{2} \mathrm{O}_{4} \mathrm{~S}_{2}{ }^{+}[\mathrm{M}+\mathrm{H}]^{+}$: 323.01603, found: 323.01544; calcd. for $\mathrm{C}_{13} \mathrm{H}_{10} \mathrm{~N}_{2} \mathrm{O}_{4} \mathrm{~S}_{2} \mathrm{Na}^{+}[\mathrm{M}+\mathrm{Na}]^{+}:$344.99797, found: 344.99737; Anal. calcd. for $\mathrm{C}_{13} \mathrm{H}_{10} \mathrm{~N}_{2} \mathrm{O}_{4} \mathrm{~S}_{2}$ (322.36): C, 48.44; H, 3.13; N, 8.69; S, 19.89. Found: C, 48.39; H, 3.18; N, 8.54; S, 19.97. 
6-Cyclopropyl-3-methyl-2,9-dioxo-2,3,6,9-tetrahydro-1H-pyrido[3', 2' : 4,5] thieno[2,3b] $[1,4]$ thiazine-8-carboxylic acid $[( \pm)-9 \mathbf{b}]$

This compound was prepared via reductive cyclization of ( \pm )- 2-[(1-carboxyethyl)thio]-7cyclopropyl-3-nitro-4-oxo-4,7-dihydrothieno[2,3-b]pyridine-5-carboxylic acid $[( \pm)-7 \mathbf{b}, 0.38$ g, 1 mmol] using stannous chloride dihydrate $(1.13 \mathrm{~g}, 5 \mathrm{mmol})$, and following the same procedure and experimental conditions described above for obtaining 9a. The title product was formed as a yellow precipitate which was collected, washed with ethanol and dried. Yield $0.26 \mathrm{~g}$ (77 \%); mp 246-247 ${ }^{\circ} \mathrm{C}$ (decomp); IR (cm $\left.{ }^{-1}\right) 3453$ (br), 3318, 3096, 3048, 1738, 1682, 1649, 1612, 1520, 1467, 1431, 1332, 1297, 1249, 1186; ${ }^{1} \mathrm{H}-\mathrm{NMR}$ (300 MHz, DMSO-d 6 ) $\delta$ 1.15/1.28 (2 m, 4H, 2H-2' / 2H-3'), 1.35 (d, $J=7$ Hz, 3H, $\mathrm{CH}_{3}$ ), 3.83 (m, 1H, H-1'), 3.85 (q, $J=7$ Hz, 1H, H-3), 8.54 (s, 1H, H-7), 9.54 (s, 1H, H-1), 14.83 (br s, $\left.\mathrm{CO}_{2} \mathrm{H}\right) ;{ }^{13} \mathrm{C}-\mathrm{NMR}\left(75 \mathrm{MHz}, \mathrm{DMSO}-\mathrm{d}_{6}\right) \delta 7.5\left(\mathrm{C}-2^{\prime} / \mathrm{C}-3\right.$ '), $15.5\left(\mathrm{CH}_{3}\right), 37.9(\mathrm{C}-3), 38.2(\mathrm{C}-$ 1'), 105.7 (C-4a), 112.0 (C-9b), 119.7 (C-8), 131.9 (C-9a), 145.9 (C-7), 152.0 (C-5a), $165.6\left(\mathrm{CO}_{2} \mathrm{H}\right)$, 165.7 (C-2), 173.7 (C-9); HRMS: calcd. for $\mathrm{C}_{14} \mathrm{H}_{13} \mathrm{~N}_{2} \mathrm{O}_{4} \mathrm{~S}_{2}{ }^{+}[\mathrm{M}+\mathrm{H}]^{+}$: 337.03168, found: 337.03114; calcd. for $\mathrm{C}_{14} \mathrm{H}_{12} \mathrm{~N}_{2} \mathrm{O}_{4} \mathrm{~S}_{2} \mathrm{Na}^{+}[\mathrm{M}+\mathrm{Na}]^{+}$: 359.01362, found: 359.01313; Anal. calcd. for $\mathrm{C}_{14} \mathrm{H}_{12} \mathrm{~N}_{2} \mathrm{O}_{4} \mathrm{~S}_{2}$ (336.39): C, 49.99; H, 3.60; N, 8.33; S, 19.06. Found: C, 49.97; H, 3.53; N, 8.42; S, 18.80.

3-(Carboxymethyl)-6-cyclopropyl-2,9-dioxo-2,3,6,9-tetrahydro-1H-pyrido-[3',2':4,5] thieno[2,3b] [1,4]-thiazine-8-carboxylic acid [ ( \pm )-9c]

This compound was prepared via reductive cyclization of ( \pm )-2-[(5-carboxy-7-cyclopropyl-3-nitro4-oxo-4,7-dihydrothieno[2,3-b]pyridin-2-yl)thio]succinic acid [( \pm )-7c, $0.43 \mathrm{~g}, 1 \mathrm{mmol}]$ using stannous chloride dihydrate $(1.13 \mathrm{~g}, 5 \mathrm{mmol})$, and following the same procedure and experimental conditions described above for the preparation of 9a. The title compound was isolated as a yellow solid which was washed with ethanol and dried. Yield $0.28 \mathrm{~g}$ (74 \%); mp 239-240 ${ }^{\circ} \mathrm{C}$ (decomp); IR ( $\mathrm{cm}^{-1}$ ) 3464 (br), 3342, 3095, 3016, 2940, 1717, 1697, 1601, 1549, 1471, 1439, 1363, 1325, 1244, 1202, 1171; ${ }^{1} \mathrm{H}-$ NMR (300 MHz, DMSO-d ${ }_{6}$ ) $\delta 1.17 / 1.27$ (2 m, 4H, 2H-2' / 2H-3'), 2.55 (dd, $J=16.6,7.8 \mathrm{~Hz}, 1 \mathrm{H}, \mathrm{H}_{\mathrm{A}^{-}}$ 3"), 2.87 (dd, $J=16.6,6.1 \mathrm{~Hz}, 1 \mathrm{H}, \mathrm{H}_{\mathrm{B}}-3^{\prime \prime}$ ), 3.84 (m, 1H, H-1'), 3.98 (dd, $J=6.1,7.8 \mathrm{~Hz}, 1 \mathrm{H}, \mathrm{H}-3$ ), 8.54 (s, $1 \mathrm{H}, \mathrm{H}-7), 9.66$ (s, $1 \mathrm{H}, \mathrm{H}-1), 12.69$ [br s, $\left.1 \mathrm{H}, \mathrm{C}\left(4^{\prime \prime}\right)-\mathrm{O}_{2} \mathrm{H}\right], 14.56$ [br s, $\left.1 \mathrm{H}, \mathrm{C}(8)-\mathrm{CO}_{2} \mathrm{H}\right] ;{ }^{13} \mathrm{C}-$ NMR (75 MHz, DMSO-d 6 ) $\delta 7.5$ (C-2'/C-3'), 33.9 (C-3"), 38.2 (C-1'), 39.5 (C-3), 106.1 (C-4a), 112.0 (C-9b), 119.8 (C-8), 132.0 (C-9a), 145.9 (C-7), 152.2 (C-5a), 164.1 (C-2), 165.7 [C(8)-CO $\left.{ }_{2} \mathrm{H}\right], 171.3$ [C(4")- $\left.\mathrm{O}_{2} \mathrm{H}\right], 173.6$ (C-9); HRMS: calcd. for $\mathrm{C}_{15} \mathrm{H}_{13} \mathrm{~N}_{2} \mathrm{O}_{6} \mathrm{~S}_{2}{ }^{+}[\mathrm{M}+\mathrm{H}]^{+}$: 381.02151, found: 381.02090; calcd. for $\mathrm{C}_{15} \mathrm{H}_{12} \mathrm{~N}_{2} \mathrm{O}_{6} \mathrm{~S}_{2} \mathrm{Na}^{+}[\mathrm{M}+\mathrm{Na}]^{+}$: 403.00345, found: 403.00280; Anal. calcd. for $\mathrm{C}_{15} \mathrm{H}_{12} \mathrm{~N}_{2} \mathrm{O}_{6} \mathrm{~S}_{2}$ (380.40): C, 47.36; H, 3.18; N, 7.36; S, 16.86. Found : C, 47.02; H, 3.17; N, 7.24; S, 16.65.

\section{Acknowledgements}

We wish to thank the Deanship of Scientific Research-The University of Jordan, Amman-Jordan for financial support.

\section{References and Notes}

1. El-Abadelah M. M.; Sabri S. S.; Al-Ashqar H. A. Thienopyridone Antibacterials. Part I. A 
Synthesis of Some 7-Alkyl-2-chloro-1,4-dihydro-1-oxothieno[2,3-b]pyridine-5-carboxylic Acids. Heterocycles 1997, 45, 255-264, and refs. cited therein.

2. El-Abadelah M. M.; Sabri S. S.; Al-Ashqar H. A.; Mion P.; Bompart J.; Calas M. Thienopyridinone Antibacterials. Part II. Synthesis and Antibacterial Activity of Some 2-Chloro-7cyclopropyl-4,7-dihydro-4-oxothieno[2,3-b]pyridine-5-carboxylic Acids. J. Phosphorus Sulfur Silicon Rel. Elem. 1998, 67, 21-29.

3. El-Abadelah M. M.; Nazer M. Z.; Okasha S. F.; Calas M.; Bompart J.; Mion P. Thienopyridinone Antibacterials: Synthesis and Antibacterial Activity of Some 7-Aryl-2-chloro-4,7-dihydro-4oxothieno[2,3-b]pyridine-5-carboxylic Acids. Eur. J. Med. Chem. 1998, 33, 33-42.

4. (a) Bompart J.; Giral L.; Malicorne G.; Puygrenier M. Synthesis and Antibacterial Activities of New 4,7-Dihydro-7-ethyl-4-oxothieno[2,3-b]pyridine-5-carboxylic Acids. Eur. J. Med. Chem. 1987, 22, 139-145, and refs. cited therein; (b) Bompart J.; Giral L.; Malicorne G.; Puygrenier M. Synthesis and Anti-bacterial Activity of 4,7-Dihydro-7-ethyl-4-oxothieno[2,3-b]pyridine-5carboxylic Acids. Eur. J. Med. Chem. 1988, 23, 457-464; (c) Giral L.; Bompart J.; Puygrenier M. Thienopyridinone Derivatives and Pharmaceutical Compositions Containing Them. Eur. Pat. Appl. EP 161,235, 1985.

5. Schreder M. E.; Erker T. Studies on the chemistry of thienoannelated O,N- and S,N-containing heterocycles. 17 Preparation of $1 \mathrm{H}$-Thieno[2,3-b][1,4]thiazine-1-carboxamides as Smooth Muscle Relaxants. J. Heterocycl. Chem. 2000, 37, 349-354.

6. Erker T.; Schreder M. E.; Studenik C. Studies on the Chemistry of Thienoannelated O,N- and S,NContaining Heterocycles. Part 19: Thieno[2,3-b][1,4]thiazines with Calcium Antagonistic and Potassium Opening Activities. Arch. Pharm. 2000, 333, 58- 62.

7. Tanaka A.; Mizuno H.; Sakurai M. Preparation of Novel Benzothiophene Derivatives and Their Use as Urokinase Inhibitors. PCT. Int. Appl. WO 9811089, 1998.

8. Kakehi A.; Ito S.; Hakui J.; Fukazawa H. Preparation of New Nitrogen-Bridged Heterocycles. 29. Reinvestigation of the Dehydrogenation Reaction of 5,5a-Dihydropyrido[2,1-c]thieno[3,2e][1,4]thiazine Derivatives. Bull. Chem. Soc. Jpn. 1992, 65, 1244-1251.

9. Al-Huniti M. H.; Zahra J. A.; El-Abadelah M. M. Heterocycles [h]Fused on 4-Oxoquinoline-3carboxylic Acid, II. A Facile Synthesis of Some 2,7-Dioxo[1,4]thiazino[2,3-h]quinoline-8carboxylic Acids. Heterocycles 2007, 71, 411-418.

Sample availability: Contact the authors.

(C) 2007 by MDPI (http://www.mdpi.org). Reproduction is permitted for noncommercial purposes. 